\title{
A Case of 2-Year-Old Child with Entero-Enteric Fistula Following Ingestion of 25 Magnets
}

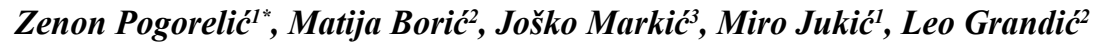 \\ ${ }^{1}$ Department of Pediatric Surgery, Split University Hospital and University of Split, School of Medicine, Split, Croatia \\ ${ }^{2}$ Department of Surgery, Split University Hospital and University of Split, School of Medicine, Split, Croatia \\ ${ }^{3}$ Department of Pediatrics, Split University Hospital and University of Split, School of Medicine, Split, Croatia \\ * Corresponding author: Department of Pediatric Surgery, Split University Hospital, Spinčićeva 1, 21000 Split, Croatia; \\ e-mail: zpogorelic@gmail.com
}

Summary: Introduction: Magnet ingestion usually does not cause serious complications, but in case of multiple magnet ingestion or ingestion of magnet with other metal it could cause intestinal obstruction, fistula formation or even perforation. Case report: We report case of intestinal obstruction and fistula formation following ingestion of 25 magnets in a 2-year-old girl. Intraoperatively omega shaped intestinal loop with fistula caused by two magnetic balls was found. Intestine trapped with magnetic balls was edematous and inflamed. Resection of intestinal segment was performed, followed by entero-enteric anastomosis. A total of 25 magnets were removed from resected intestine. Conclusion: Single magnet ingestion is treated as non-magnetic foreign body. Multiple magnet ingestion should be closely monitored and surgical approach could be the best option to prevent or to cure its complications.

Keywords: Ingestion; Magnetic foreign body; Multiple magnets; Intestinal fistula; Children

\section{Introduction}

Ingested foreign bodies are a common problem throughout the world. More than three quarters of foreign body ingestions occur in children (1). In $90 \%$ of cases swallowed foreign bodies pass harmlessly through the gastrointestinal tract. However, around 10-20\% of foreign body ingestions require endoscopic removal, and around $1 \%$ will require surgical intervention (2). Some of foreign bodies require special considerations, such as batteries and magnets $(3,4)$. In the last decade the incidence of ingested magnets has grown due to increased use of magnetic toys (4). Single magnet ingestion usually ends spontaneously and do not cause serious complications that require surgical intervention but in case of multiple magnet ingestion or ingestion of magnet with other metal it could cause intestinal obstruction, fistula formation or even perforation $(5,6)$.

Here we present a case of intestinal obstruction and fistula formation following ingestion of 25 magnets in a 2-year-old girl.

\section{Case report}

A previously healthy 2-year-old girl presented to our surgical emergency department, because of ingestion of several small, spherical magnets earlier that morning. Parents did not see her swallowing those magnets, but they suspected it, and estimated number of around 20-25 magnets. Physical examination was unremarkable; abdomen was soft and flat, with no signs of peritoneal irritation. Initial abdominal $\mathrm{X}$-ray revealed a metallic foreign body $\mathrm{V}$-shaped necklace formation in middle abdomen (Fig. 1). Based on unchanged clinical status, with no fever, no lethargy or abdominal pain, with all vital signs being normal, the patient was discharged from hospital. The parents got instructions to monitor her stool, and got instructions and description of signs of possible complications. The patient was on repeated follow-ups where unchanged vital signs were found. Repeated abdominal X-ray revealed aboral progression of magnets, but still not reaching the colon (Fig. 2). Nine days following foreign body ingestion, the child was brought back to hospital because she had refused any food. Examination showed that the patient appeared dehydrated with distended, but soft abdomen, with no signs of peritoneal irritation. Following $\mathrm{X}$-ray and laboratory test, along with discussion of potential risks and benefits of surgical treatment, exploratory laparotomy through lower midline incision was performed. Around $100 \mathrm{~cm}$ aboral to the ligament of Treitz omega shaped intestinal loop with fistula caused by two magnetic balls was found (Fig. 3). Intestine trapped into magnetic balls was edematous and inflamed. Resection of intestinal segment was performed, followed by entero-enteric anastomosis. A total of 25 magnets were removed from resected intestine (Fig. 4). After the surgery the child was admitted to De- 


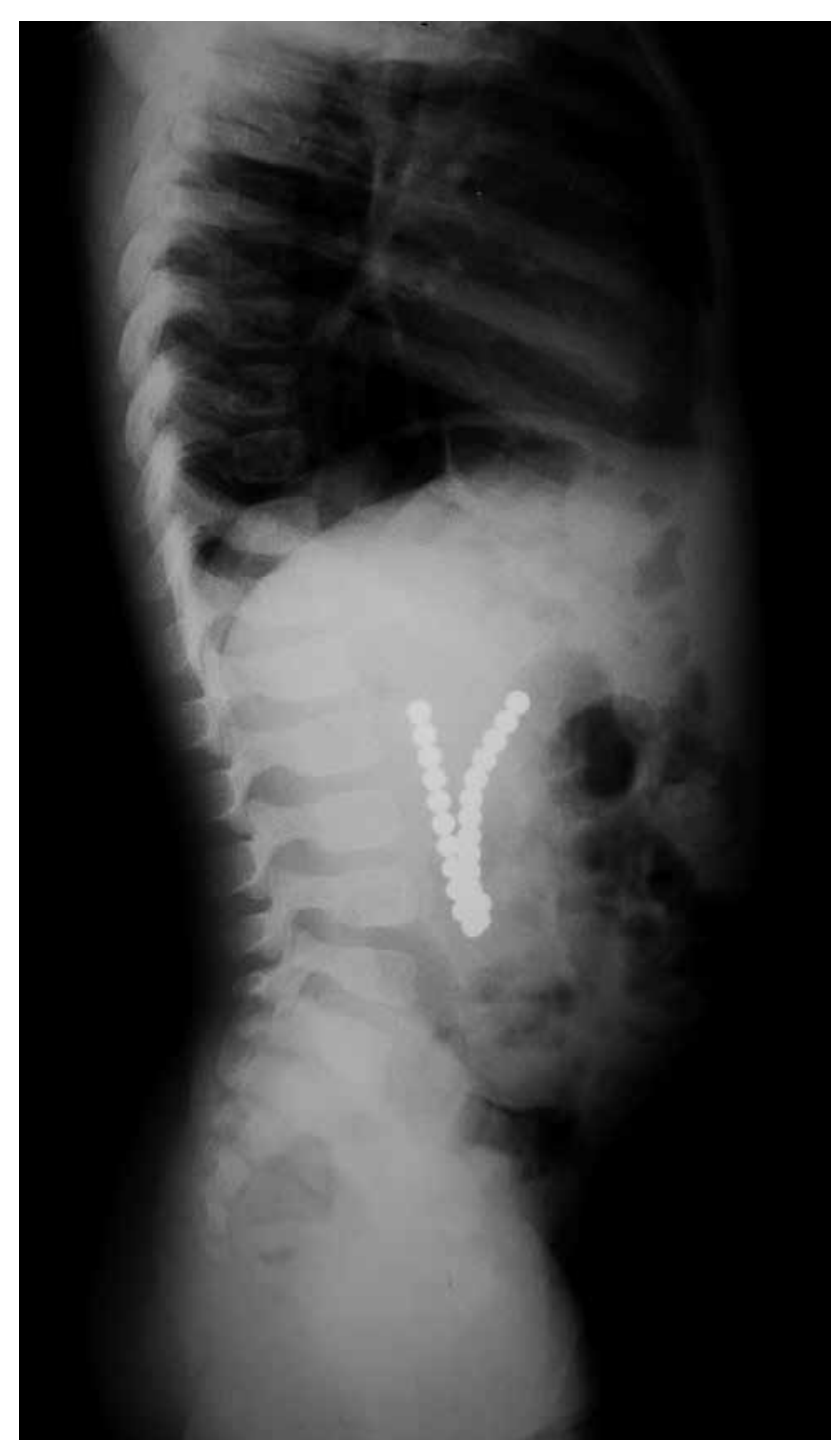

Fig. 1: Initial abdominal X-ray - a metallic foreign body: V-shaped necklace formation in middle abdomen.

partment of pediatric surgery. The patient recovered well postoperatively and she was discharged home on the 5 th postoperative day. At regular follow ups no complications were recorded.

\section{Discussion}

Ingestion of foreign bodies is a common pediatric problem, with more than 100000 cases annually. The most common ingested foreign bodies are coins, toys, batteries, bones or food. Magnets are increasingly ingested (7). Magnets have evolved rapidly in the past three decades. Neodymium magnets were created in 1980s and have much stronger adherence than previously used magnets (8). US

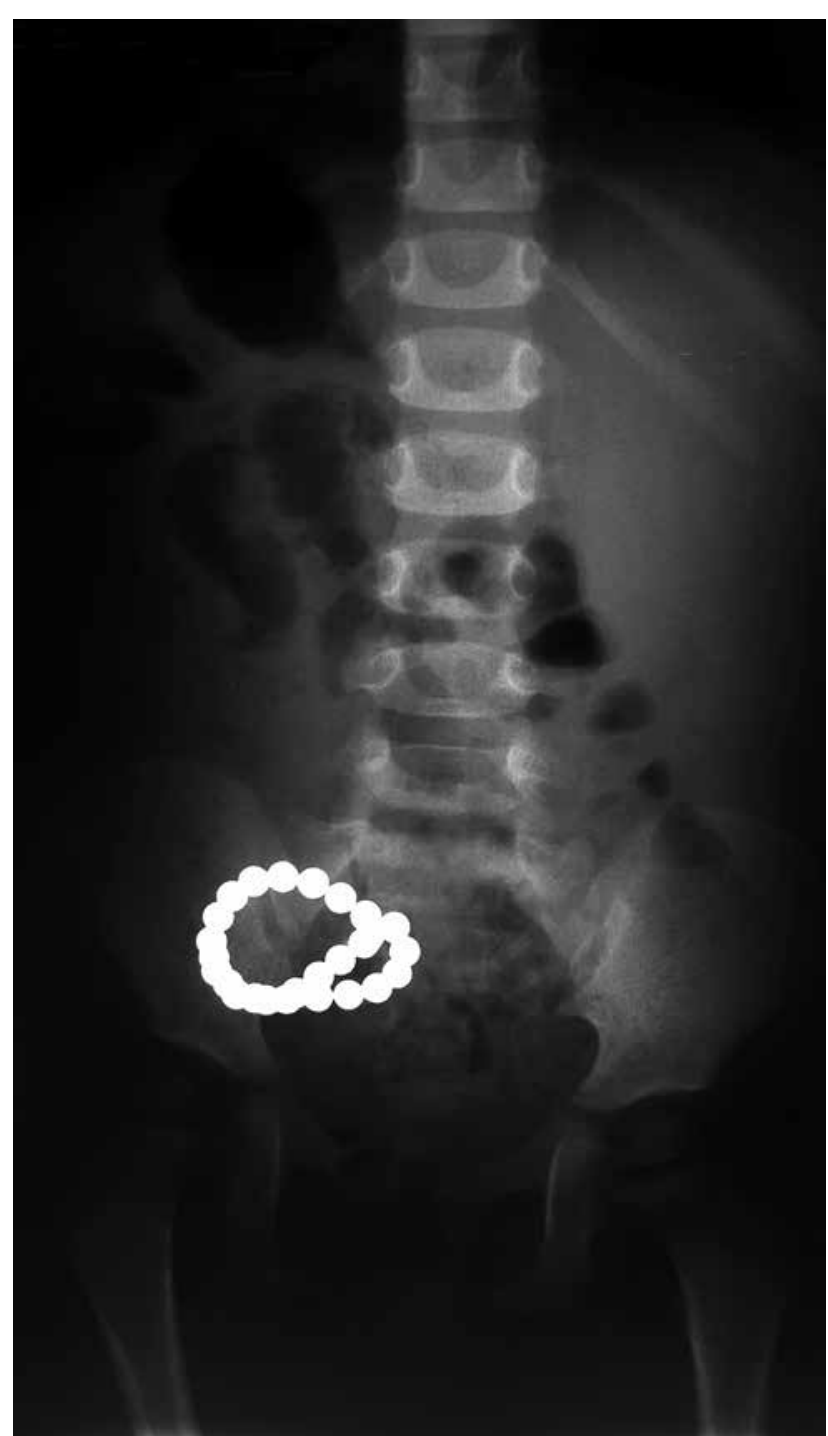

Fig. 2: Abdominal X-ray on 5th day after ingestion of foreign body - aboral progression of magnets.

National Electronic Injury Surveillance System described 8.5 times increase of possible ingestion of magnets in underage children in last decade (9). Thus Canadian and USA healthcare institutions have intention to regulate laws to keep strong magnets out of the hands of children (10).

Single magnet ingestion is like swallowing any nonmagnetic foreign body, while multiple magnets can cause serious gastrointestinal complications like entero-enteric fistula formation, bowel with associated perforation, peritonitis, and bowel ischemia or necrosis $(11,12)$.

In our case magnets were beyond ligament of Treitz and proximal to the terminal ileum. Management of asymptomatic patients in that descripted position is more controversial than in other gastrointestinal part where foreign body could 


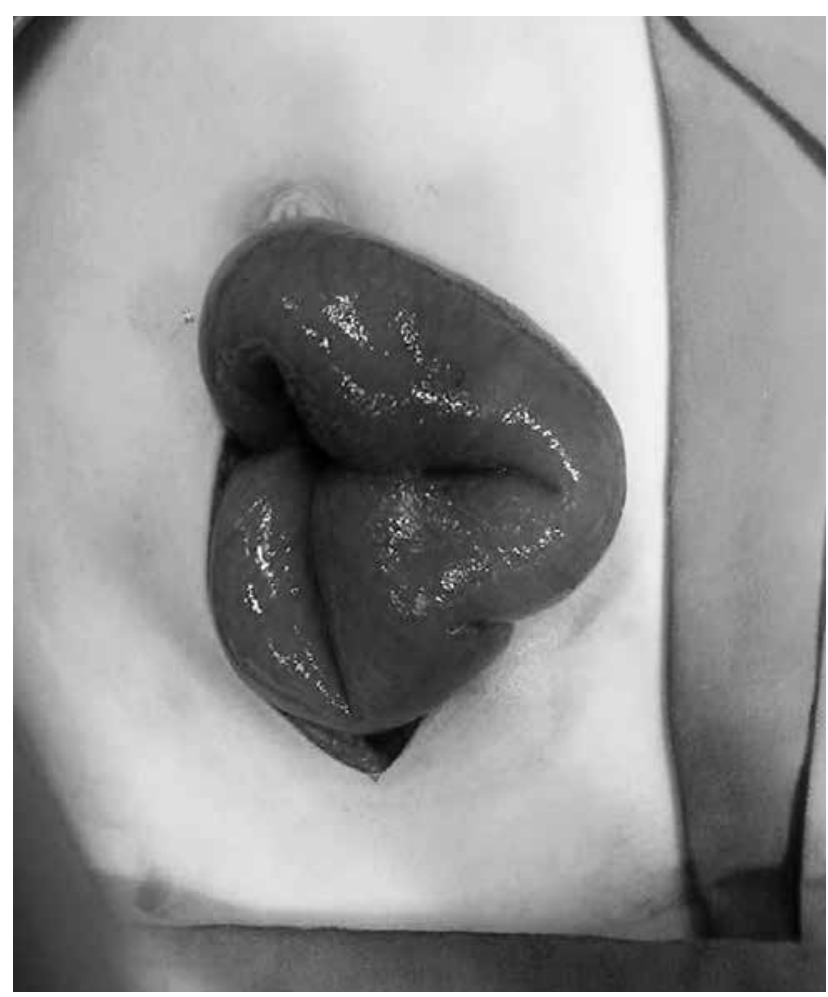

Fig. 3: Intraoperative findings - omega shaped intestinal loop with fistula caused by magnetic balls.

be reached either by esophagogastroduodenoscopy or colonoscopy. Laparotomy and laparoscopy interventions required in case of intermediate position of magnets in gastrointestinal tract increase morbidity and mortality (13). Therefore, conservative therapy should be observed as possible therapeutic option. The consensus of North American Society for Pediatric Gastroenterology, Hepatology, and Nutrition experts suggest conservative approach only in case of direct patient observation (11). Prevention is crucial for this rapidly increasing problem (1). Awareness of possible magnet ingestion as well as potential complications of it, leads to reduced time from ingestion to diagnosis and from diagnosis to final solution.

\section{Conclusion}

It is important to differentiate single from multiple magnet ingestion. Single magnet ingestion is treated as nonmagnetic foreign body. Multiple magnet ingestion should be

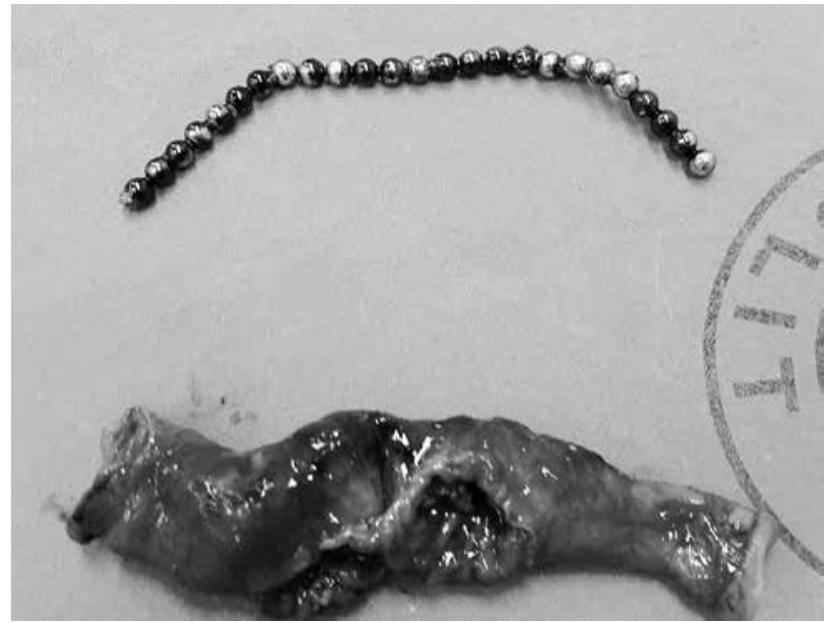

Fig. 4: Resected speciemen - intestinal segment with fistula and 25 removed magnets.

closely monitored and surgical approach could be the best option to prevent or to cure its complications.

\section{References}

1. George AT, Motiwale S. Magnets, children and the bowel: a dangerous attraction? World J Gastroenterol 2012; 18: 5324-5328.

2. Eisen GM, Baron TH, Dominitz JA, et al. Guideline for the management of ingested foreign bodies. Gastrointest Endosc 2002; 55: 802-806.

3. Hussain SZ, Bousvaros A, Gilger M, et al. Management of ingested magnets in children. J Pediatr Gastroenterol Nutr 2012; 55: 239-242.

4. Tavarez MM, Saladino RA, Gaines BA, et al. Prevalence, clinical features and management of pediatric magnetic foreign body ingestions. J Emerg Med 2013, 44: 261-268.

5. Hernandez Anselmi E, Gutierrez San Roman C, Barrios Fontoba JE, et al. Intestinal perforation caused by magnetic toys. J Pediatr Surg 2007; 42: E13-6.

6. Othman MY, Srihari S. Multiple magnet ingestion: The attractive hazardst. Med J Malaysia 2016; 71:211-212.

7. Kay M, Wyllie R. Pediatric foreign bodies and their management. Curr Gastroenterol Rep 2005; 7: 212-218.

8. Sahin C, Alver D, Gulcin N, et al. A rare cause of intestinal perforation: ingestion of magnet. World J Pediatr 2010; 6: 369-371.

9. Abbas MI, Oliva-Hemker M, Choi J, et al. Magnet ingestions in children presenting to US emergency departments, 2002-2011. J Pediatr Gastroenterol Nutr 2013; 57: $18-22$

10. Rosenfield D, Strickland M, Fecteau A. Magnet ingestion by a 3-year-old boy. CMAJ 2013; 185: 972-974.

11. Kramer RE, Lerner DG, Lin T, et al. Management of ingested foreign bodies in children: a clinical report of the NASPGHAN Endoscopy Committee. J Pediatr Gastroenterol Nutr 2015; 60: 562-574.

12. Naji H, Isacson D, Svensson JF, et al. Bowel injuries caused by ingestion of multiple magnets in children: a growing hazard. Pediatr Surg Int 2012; 28: 367-734.

13. Butterworth J, Feltis B. Toy magnet ingestion in children: revising the algorithm. J Pediatr Surg 2007; 42: e3-5.

Received: $30 / 11 / 2016$

Accepted: 12/12/2016 